\title{
Low-fat and low-protein diets are associated with hearing discomfort among the elderly of Korea
}

\author{
So Young $\mathrm{Kim}^{1}$, Songyong $\mathrm{Sim}^{2}$, Hyung-Jong $\mathrm{Kim}^{3}$ and Hyo Geun $\mathrm{Choi}^{3 *}$ \\ ${ }^{1}$ Department of Otorbinolaryngology-Head E Neck Surgery and Cancer Research Institute, Seoul National University College \\ of Medicine, Seoul, 110-744 Korea \\ ${ }^{2}$ Department of Statistics, Hallym University, Chuncheon, 200-702 Korea \\ ${ }^{3}$ Department of Otorhinolaryngology-Head \& Neck Surgery, Hallym University Sacred Heart Hospital, Anyang, 431-796 Korea
}

(Submitted 2 April 2015 - Final revision received 2 June 2015 - Accepted 10 August 2015 - First published online 21 September 2015 )

\section{Abstract}

Research on the effect of low-fat intake on hearing is limited. This study aimed to elucidate the relationship between fat, carbohydrate and protein intake and the presence of hearing discomfort among the elderly. The Korean National Health and Nutrition Examination Survey was conducted from 2009 through to 2012. A total of 4615 participants ranging in age from 60 to 80 years underwent a pure-tone audiometric evaluation, a physical examination and a nutritional survey. The associations between the participants' hearing thresholds and their protein/ fat/carbohydrate intake/total energy intake were analysed using simple and multiple regression models with complex sampling adjusted for confounding factors, such as BMI, income level, smoking status and a history of hypertension, hyperlipidaemia and diabetes. Low fat and protein intakes were associated with hearing discomfort (OR 0.82, 95\% CI 0.71, 0.96, $P=0.011$; OR 0.81, 95\% CI 0.67, 0.96, $P=0.017$, respectively). This study revealed that low fat and protein intakes are associated with hearing discomfort in the elderly Korean population.

Key words: Diet: Asia: Dietary fat: Protein: Carbohydrates: Elderly

A trend towards increasing dietary fat intake has been noted worldwide. The fat:energy ratio is above the minimum recommended value of $15 \%$ in some developed countries ${ }^{(1,2)}$. High fat contributes to the increasing prevalence of obesity. In America, approximately $70 \%$ of people aged 65 years or older are overweight (BMI, $\mathrm{kg} / \mathrm{m}^{2} \geq 25$ ) or obese (BMI $\geq 30$ ) as per the 2007-2012 National Health and Nutrition Examination Survey (NHANES) $^{(3)}$. Increased fat intake is associated with metabolic risk factors ${ }^{(4)}$. Particularly in the elderly population, greater attention to fat intake is warranted. Elderly people differ from younger populations with regard to metabolism and fat distribution. Older individuals have more stored body fat and less lean mass compared with young adults ${ }^{(5)}$. Moreover, because of their increased insulin resistance, elderly people have a greater proportion of body fat in the intrahepatic, intramuscular and intra-abdominal compartments ${ }^{(5)}$. These changes lead to poor metabolic outcomes for older people.

However, a low-fat diet may not always be beneficial for health ${ }^{(6)}$. Previous evidence has suggested that some types of fat, such as fat from tree nuts and extra-virgin olive oil, reduce cardiovascular events and risk factors, atrial fibrillation, peripheral artery disease, type 2 diabetes and the metabolic syndrome ${ }^{(7)}$. Although there is some controversy surrounding the effects of fat ${ }^{(8,9)}$, recent studies have suggested that diets that are high in certain fats, including some MUFA and PUFA, and that contain adequate amounts of essential fatty acids are associated with reduced risk for CVD, metabolic diseases, cancer and depression ${ }^{(10,11)}$. These findings of the benefits of fat raised questions about the effects of the amount of fat intake on health outcomes $^{(10)}$.

Hearing discomfort is one of the most common degenerative disorders among elderly people and is generally irreversible. Several studies have focused on modifiable factors associated with hearing discomfort, particularly nutritional factors. Previous studies have demonstrated that a diet rich in folate, fibre and vitamins $\mathrm{A}, \mathrm{E}$ and $\mathrm{C}$ is beneficial for alleviating age-related hearing discomfort $^{(12-14)}$. Generally, a high-fat diet is associated with hearing discomfort. Many studies have demonstrated that increased dietary intake of fats, particularly cholesterol and SFA, accelerates the development of age-related hearing discomfort ${ }^{(15,16)}$. However, most of these studies did not consider the appropriate proportional intake of fat and other nutritional factors and were based on populations with high dietary fat intakes. Previous studies reported conflicting results concerning the effects of hypercholesterolaemia on hearing. Some studies found that hyperlipidaemia was an aetiological factor in hearing

Abbreviation: KNHANES, Korean National Health and Nutrition Examination Survey.

* Corresponding author: H. G. Choi, fax +82 31386 3860, email pupen@naver.com 
discomfort ${ }^{(15,16)}$; however, other studies suggested no difference between the lipid profiles of the hearing discomfort and normal-hearing groups. These studies reported that hearing thresholds were significantly enhanced in the high-cholesterol group $^{(17,18)}$. In addition, the dietary intake of some types of fats, such as $n$-3 PUFA, is associated with hearing preservation ${ }^{(19)}$

In the adult Korean population, the proportion of dietary fat has also significantly increased from $18.5 \%$ in 1998 to $20.3 \%$ in 2012, which reflects the increasing trend towards westernised nutritional intakes ${ }^{(20)}$. Although a trend towards increasing fat intake as a result of the rapidly increasing consumption of westernised foods, such as fried chicken, pizza and hamburgers, has been observed, a rice-based diet is predominant in the Korean population, particularly among the elderly. Given this dietary characteristic, fat intake of the Korean elderly population may be reduced compared with the dietary reference intake level ${ }^{(21)}$. The proportions of dietary carbohydrates and protein have remained relatively stable, between 65 and $67 \%$ and approximately $15 \%$, respectively, from 1998 through to $2012^{(20)}$.

To date, little information regarding the effect of the proportional intakes of fat, carbohydrates and protein on the hearing abilities of Asian populations is available. In this study, we investigated the relationships between nutritional intake and hearing in the elderly. The primary outcome of the present study was to evaluate the relation between total energy/protein/fat/ carbohydrate intake and the presence of hearing discomfort. We also analysed the association of the protein/fat/carbohydrate intakes with hearing discomfort at specific frequencies $(500,1000$, 3000,4000 and $6000 \mathrm{~Hz}$ ). To exclude other factors associated with hearing, we applied a multiple logistic regression analysis with complex sampling. Moreover, to exclude the influence of total energy intake, we calculated the proportional intakes of fat and carbohydrate compared with the total energy intake. The protein and total energy intakes were compared with the age- and sexmatched recommended dietary intake for the Korean population. These standardisations enabled us to further exclude the confounding effects of age, sex, BMI, income, smoking status and the underlying disease status.

\section{Methods}

\section{Study population and data collection}

This study was approved by the institutional review board of the Korean Centers for Disease Control and Prevention. Written informed consent was obtained from all participants before commencement of the study.

This cross-sectional study used data from the Korean National Health and Nutrition Examination Survey (KNHANES) and included the entire national population. The survey included a health interview, a nutritional survey and physical examinations. Statistical methods were applied on the basis of the sampling design using adjusted weighted values. The KNHANES data collected by the Centers for Disease Control and Prevention of Korea from 2009 to 2012 were analysed. Each year, 192 districts were selected by a panel. A total of twenty households from each selected district were identified to obtain a sample that was reflective of the entire Korean population. The surveys were obtained from the civilian, non-institutionalised South Korean population using a stratified, multistage, clustered sampling method based on the national census data. The sample was weighted by statisticians, who performed post-stratification analyses and accounted for the non-response rate and for extreme values. Among the selected candidate population, $80 \cdot 8 \%$ of individuals participated in the KNHANES from 2009 to 2012.

As a result, a total 36067 people were sampled; of these, the following participants were excluded from this study: participants aged under 60 years or over 80 years (27958 participants); participants who did not undergo audiometric testing or a physical examination or who had an abnormal tympanic membrane (TM; 3020 participants); participants who had not completed the nutritional survey (331 participants); participants who had incomplete data on BMI $\left(\mathrm{kg} / \mathrm{m}^{2}\right)$, income level and smoking status; and participants who had a history of hypertension or hyperlipidaemia, and the presence of diabetes mellitus (143 participants). Finally, 4615 participants (2048 males and 2567 females) were included in this study (Fig. 1). To focus on the elderly population, the survey participants ranged in age from 60 to 80 years. To avoid survival effects, we excluded subjects who were $\geq 80$ years of age.

\section{Survey}

Food intake data were obtained by trained staff using the complete $24 \mathrm{~h}$ recall method. The answers obtained on certain days, such as holidays or weekends, were not included. The total energy $(\mathrm{kJ} / \mathrm{d}(\mathrm{kcal} / \mathrm{d}))$ and total protein $(\mathrm{g} / \mathrm{d})$ intake were calculated by referencing the nutrient contents of foods described in the Korean Food Composition Table ${ }^{(22)}$. The intake amount was compared with the recommended intakes for Korean people ${ }^{(23)}$. Therefore, the proportional total energy

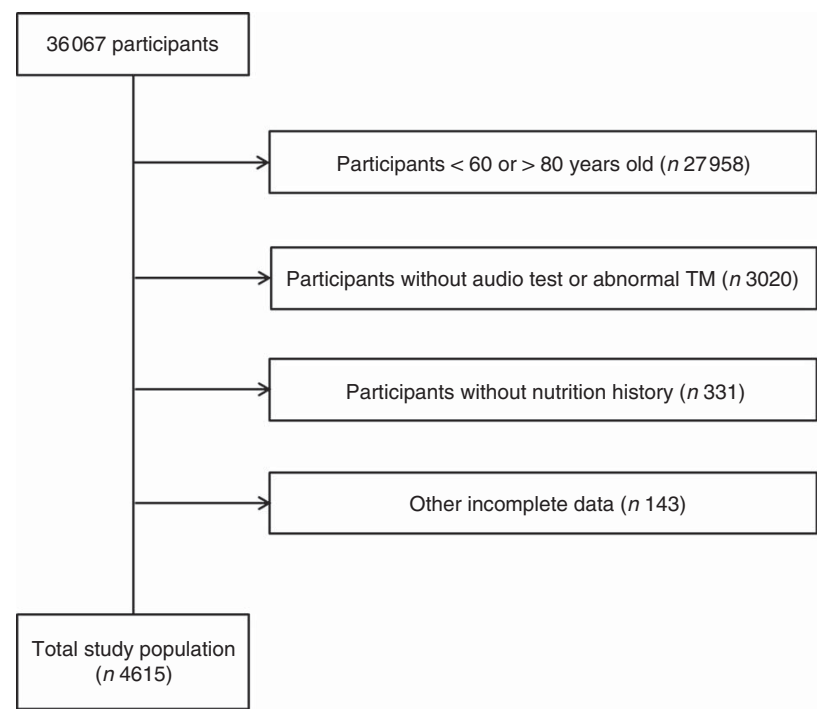

Fig. 1. A schematic illustration of participant selection in the present study. Among a total of 36067 participants, participants aged 60 to 80 years who underwent audiological testing, showed normal tympanic membrane (TM) findings and provided a nutritional history were selected for this study. The data on the 4615 participants from whom complete data were obtained were analysed. 
and total protein intakes of each participant were calculated by adjusting for age and sex. For example, the proportion of total intake energy $(\%)=$ total energy intake/age- and sex-matched recommended total energy intake. Unlike for other nutritional components, the balance of proteins, fats and carbohydrates is the most important aspect of fat and carbohydrate intake measurements ${ }^{(23)}$. Thus, fat and carbohydrate intake were measured as the proportion of the total energy intake rather than the proportion of age- and sex-matched recommended intake $e^{(24)}$. The fat and carbohydrate intakes were measured using the following methods: fat energy intake/total energy intake (\%) and carbohydrate energy intake/total energy intake (\%). According to each nutrient intake compared with the average values, the subjects were divided into high and low groups (Table 1). The reference values for total energy, protein, fat and carbohydrate distributions were 100,100,12,74 and 25, respectively.

In this study, trained otorhinolaryngologists examined the TM of all the participants using a $4 \mathrm{~mm}$-diameter, $0^{\circ}$-angled rigid endoscope (Xion GmbH). Participants with an abnormal TM were excluded from this study. Pure-tone audiometry was performed in a soundproof booth using an automatic audiometer (SA 203; Entomed) to determine the air-conduction hearing thresholds at 500, 1000, 2000, 3000, 4000 and $6000 \mathrm{~Hz}$ in both ears. Hearing discomfort was defined as a hearing discomfort exceeding an average of $25 \mathrm{~dB}$ on pure-tone audiometry at 500,1000, 2000, and $4000 \mathrm{~Hz}^{(25)}$. If the right and left ear hearing thresholds differed, the worse hearing threshold was selected.

By dividing the household income by the square root of the number of household members, the monthly income was divided into four quartiles from top to bottom: lowest, low middle, upper middle and highest. Smoking status was divided into the two following categories: smoked less than five packs (100 cigarettes) in a lifetime and smoked five or more packs in a lifetime. Diagnoses of the possible comorbidities of hearing discomfort, including hypertension, hyperlipidaemia and diabetes mellitus, were documented using the questionnaire. The participants were considered positive for these comorbidities if they had been diagnosed by a medical doctor.

\section{Statistical analysis}

The general characteristics of the participants were calculated by adjusting the recommended weighted values based on the KNHANES data (Table 1).

The OR of each nutritional factor for the hearing discomfort was calculated. Simple logistic regression analysis with complex sampling (unadjusted) was performed. Multiple logistic regression analysis with complex sampling adjusted for age and sex was performed (model 1). Multiple logistic regression analysis with complex sampling adjusted for age, sex, BMI, income, smoking status and history of hypertension, hyperlipidaemia and diabetes mellitus was performed (model 2; Table 2).

The OR for the hearing thresholds (dB) at 500, 1000, 2000, 3000,4000 and $6000 \mathrm{~Hz}$ were examined according to the fat and carbohydrate proportions of the total energy intake and protein intake, using multiple logistic regression analysis with complex
Table 1. General characteristics of participants* (Means and standard deviations; numbers and percentages)

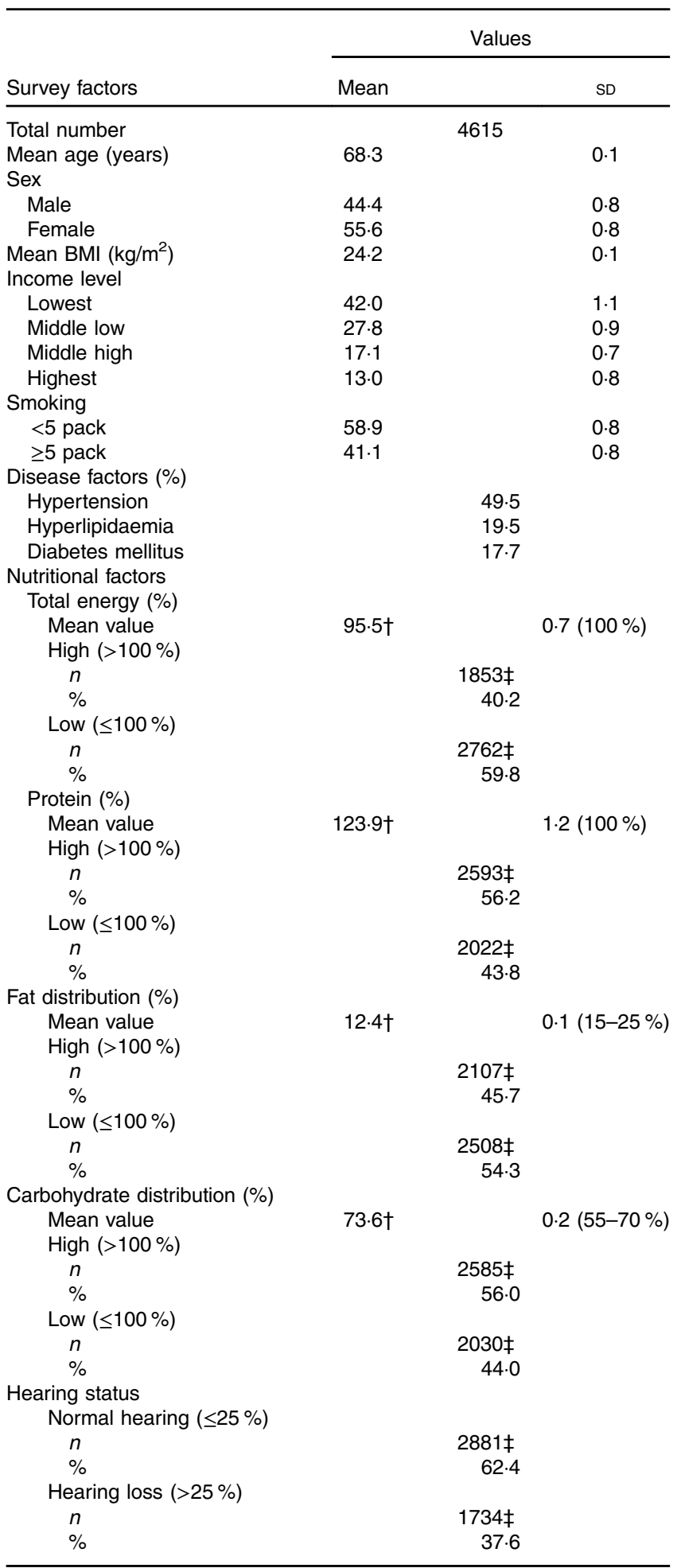

* Mean values and rates of each factor were adjusted with recommended weighted values. $\dagger$ Dietary reference of the recommended intakes for Koreans (KDRI). $\ddagger$ Number of subjects per group.

sampling adjusted for age, sex, BMI, income, smoking status and history of hypertension, hyperlipidaemia and diabetes mellitus (Table 3). 
Table 2. Odds ratios of nutritional factors for hearing discomfort ( $>25 \mathrm{~dB}$ ) by multiple logistic regression analysis with complex sampling (Odds ratios and $95 \%$ confidence intervals)

\begin{tabular}{|c|c|c|c|}
\hline Nutritional factors & OR & $95 \% \mathrm{Cl}$ & $P$ \\
\hline \multicolumn{4}{|l|}{ Total energy } \\
\hline Unadjusted & 0.85 & $0.74,0.97$ & $0.034^{*}$ \\
\hline Model $1 \dagger$ & 0.87 & $0.75,1.01$ & 0.073 \\
\hline Model $2 \ddagger$ & 0.89 & $0.77,1.04$ & 0.147 \\
\hline \multicolumn{4}{|l|}{ Protein intake } \\
\hline Unadjusted & 0.72 & $0.61,0.85$ & $<0.001^{*}$ \\
\hline Model $1 \dagger$ & 0.77 & $0.65,0.92$ & $0.004^{*}$ \\
\hline Model $2 \ddagger$ & 0.81 & $0.67,0.96$ & $0.017^{*}$ \\
\hline \multicolumn{4}{|c|}{ Fat energy/total energy } \\
\hline Unadjusted & 0.74 & $0.64,0.85$ & $<0.001^{*}$ \\
\hline Model $1 \dagger$ & 0.79 & $0.68,0.92$ & $0.002^{*}$ \\
\hline Model $2 \ddagger$ & 0.82 & $0.71,0.96$ & $0.011^{*}$ \\
\hline \multicolumn{4}{|c|}{ Carbohydrate energy/total energy } \\
\hline Unadjusted & $1 \cdot 15$ & $0.99,1.33$ & 0.071 \\
\hline Model 1† & $1 \cdot 18$ & $1.00,1.39$ & $0.047^{*}$ \\
\hline Model $2 \ddagger$ & 1.15 & $0.98,1.35$ & 0.094 \\
\hline
\end{tabular}

$\dagger$ Adjusted for age and sex.

$\ddagger$ Adjusted for age, sex, BMI, income, smoking, history of hypertension and hyperlipidaemia, and diabetes mellitus.

* Significant at $P<0.05$.

Table 3. Odds ratios of the hearing discomfort (hearing threshold $>25 \mathrm{~dB}$ hearing loss) at each $500,1000,2000,3000,4000$ and $6000 \mathrm{~Hz}$ in high-intake groups for fat, protein and carbohydrate by multiple logistic regression analysis with complex samplingt

(Odds ratios and $95 \%$ confidence intervals)

\begin{tabular}{llll}
\hline & OR & $95 \% \mathrm{Cl}$ & $P$ \\
\hline Fat energy/total energy $(\mathrm{Hz})$ & & & \\
500 & 0.94 & $0.80,1.09$ & 0.390 \\
1000 & 0.83 & $0.71,0.96$ & $0.010^{*}$ \\
2000 & 0.92 & $0.80,1.07$ & 0.292 \\
3000 & 0.82 & $0.70,0.97$ & $0.017^{*}$ \\
4000 & 0.80 & $0.67,0.96$ & $0.017^{*}$ \\
6000 & 0.94 & $0.71,1.23$ & 0.625 \\
Carbohydrate energy/total energy (Hz) & & & \\
500 & 1.09 & $0.94,1.27$ & 0.260 \\
1000 & 1.16 & $0.99,1.35$ & 0.065 \\
2000 & 1.05 & $0.90,1.22$ & 0.559 \\
3000 & 1.20 & $1.01,1.42$ & $0.034^{*}$ \\
4000 & 1.26 & $1.06,1.52$ & $0.011^{*}$ \\
6000 & 0.99 & $0.76,1.30$ & 0.950 \\
Protein intake & & & \\
500 & 0.86 & $0.72,1.03$ & 0.101 \\
1000 & 0.82 & $0.70,0.96$ & $0.015^{*}$ \\
2000 & 0.88 & $0.74,1.04$ & 0.128 \\
3000 & 0.94 & $0.78,1.12$ & 0.457 \\
4000 & 0.91 & $0.74,1.11$ & 0.352 \\
6000 & 0.85 & $0.64,1.14$ & 0.28 \\
\hline
\end{tabular}

$\dagger$ Analysis was adjusted for age, sex, BMI, income, smoking, history of hypertension and hyperlipidaemia, and diabetes mellitus.

* Significant at $P<0.05$.

Two-tailed analyses were conducted, and $P$ values $<0.05$ were considered significant. In addition, 95\% CI was calculated. The weighted values recommended by the KNHANES were applied, and all of the results were stated as weighted values. The statistical analyses were performed using SPSS version 21.0 (IBM).

\section{Results}

The demographic and nutritional data on the total study population are summarised in Table 1 . The mean age of the participants was $68 \cdot 3$ (SD 0.1) years, and their mean BMI $(24 \cdot 2$ (sD $0 \cdot 1) \mathrm{kg} / \mathrm{m}^{2}$ ) was within the normal range. Their mean fat (12.4 (SD 0.1) \%) and total energy (73.6 (SD 0.2) \%) intakes were lower than the recommended values (15-25 and 55-70\%, respectively). In contrast, their mean protein (123.9 (SD 1.2)\%) and carbohydrate $(73.6$ (SD 0.2$) \%$ ) intakes were higher than the recommended values (55-70\% for carbohydrates).

Multiple logistic regression analysis revealed an association between the mean hearing thresholds and the nutritional intake (Table 2). The fat intake was negatively correlated with the mean hearing thresholds; the unadjusted OR was 0.74 (95\% CI $0.64,0.85, P<0.001)$. When adjusted for age and sex, the OR was 0.79 (95\% CI $0.68,0.92, P=0.002)$, and the significance of the correlation was maintained even when the results were adjusted for all of the retrieved variables (OR 0.82, 95\% CI 0.71, $0.96, P=0 \cdot 011$; Table 2). In contrast, the carbohydrate intake exhibited a positive correlation with the mean hearing thresholds in model 1 . The unadjusted OR for age and sex was $1 \cdot 18$ (95\% CI 1.00, 1.39, $P=0.047$ ); however, when adjusted for all of the retrieved variables, the association was NS.

The total energy intake exhibited a significant correlation with the mean hearing thresholds only in the unadjusted model (OR $0.85,95 \% \mathrm{CI} 0.74,0.97, P=0.034$ ). The protein intake was significantly associated with the mean hearing thresholds in the unadjusted model (OR 0.72, 95\% CI 0.61, 0.85, $P<0.001$ ). When adjusted for other factors in the study, the OR was $0 \cdot 81$ (95\% CI 0.67, 0.96, $P=0 \cdot 017$ ).

Next, we focused on fat, carbohydrate and protein intake and evaluated their contributions to the hearing thresholds at each frequency. Each nutrient intake was significantly associated with the hearing threshold at some of the measured frequencies (Table 3). High-fat intake exhibited statistically significant correlation with decreased hearing thresholds, with adjusted ORs in the range of 0.80 at $4000 \mathrm{~Hz}(95 \% \mathrm{CI} 0.67,0.96, P=0.010)$. The carbohydrate intake was significantly correlated with the hearing thresholds at $3000 \mathrm{~Hz}(\mathrm{OR} 1 \cdot 20,95 \%$ CI 1.01, 1.42, $P=0 \cdot 034)$ and $4000 \mathrm{~Hz}$ (OR 1.26, $95 \%$ CI 1·06, 1·52, $P=0 \cdot 11$ ). The protein intake was significantly associated with the hearing threshold at $1000 \mathrm{~Hz}$ (OR 0.82, $95 \%$ CI $0 \cdot 70,0 \cdot 96, P=0 \cdot 015$ ).

\section{Discussion}

Our results indicate that low fat and protein intakes are correlated with hearing discomfort in the elderly Koreans. To our knowledge, this is the first report on low-fat intake and its effects on hearing. Moreover, our data were based on pure-tone audiometric findings, which were used to analyse the relationships between fat, carbohydrate and protein intake and the hearing thresholds at each frequency.

Although a high-fat diet can have harmful effects on health, such as increasing the risk for CVD and other metabolic syndromes, fat is essential for cellular membrane functions given its effects on lipid rafts; furthermore, fat regulates the metabolism of many fat-soluble vitamins, modulates ion-channel function and promotes brain development and function ${ }^{(26,27)}$. All of these fat-related functions are associated with hearing preservation. For example, vitamins $\mathrm{A}$ and $\mathrm{E}$ are associated with hearing 
preservation $^{(14)}$. Because vitamins $\mathrm{A}$ and $\mathrm{E}$ are lipid soluble, insufficient fat intake may hinder their metabolism and function, consequently impairing hearing sensitivity.

In addition, adipose tissue functions as a complex endocrine organ and is a major source of numerous adipokines. Adiponectin is synthesised exclusively in adipose tissue; its synthesis is inhibited by obesity-induced insulin resistance and oxidative stress in adipose tissue ${ }^{(28)}$. A low adiponectin level is associated with hearing discomfort ${ }^{(29)}$. Therefore, we hypothesise that a low-fat diet that leads to insufficient adiponectin production may not be good for hearing. Body fat has several protective effects, such as providing a rich metabolic reserve, reducing the activation of the sympathetic nervous system and lowering the levels of circulating hazardous materials, such as natriuretic peptides ${ }^{(30)}$. These protective effects of body fat may also have beneficial effects on the auditory system.

Various types of fats are related to hearing preservation; however, the mechanisms have not been well defined. The PUFA HDL-cholesterol has beneficial effects on hearing preservation ${ }^{(19)}$. Insufficient levels of PUFA are associated with neuropsychiatric diseases, such as major depression, bipolar disorder, schizophrenia, Alzheimer's disease and attention-deficit hyperactivity disorder ${ }^{(11)}$. These psychiatric disorders are associated with hearing discomfort ${ }^{(31)}$. Eicosapentaenoic and docosahexaenoic acids have been reported to have a cardioprotective function ${ }^{(32)}$. CVD have been suggested to be associated with cochlear impairment in the elderly ${ }^{(33)}$. By reducing the vascular risk factors that impair the cochlear blood supply, these types of fats may have a role in hearing preservation. Although we did not analyse the intake of specific types of fat, the negative correlation between fat intake and the hearing thresholds could be explained by the sum of the above-mentioned beneficial functions of fat. Further studies to elucidate the effects of each type of fat on hearing are warranted.

In the current study, the carbohydrate intake (73.6 (SD 0.2)\%) was somewhat increased compared with the recommended value, which is approximately $55-70 \%$ depending on age and sex ${ }^{(24)}$. Although statistical significance was only obtained with model 1 (adjusted for age and sex) for the mean hearing threshold (Table 2) and at the specific frequencies of 3000 and $4000 \mathrm{~Hz}$ (Table 3), the detrimental effects of high carbohydrate intake on hearing observed in our study were comparable to that observed in previous studies, which suggest that hyperglycaemia can cause microvascular injuries that result in increased blood viscosity and embolic or thrombotic events in the vessels of the auditory system $^{(34)}$. Previous studies showed that type 2 diabetes mellitus is related to hearing discomfort through angiopathy, neuropathy, oxidative stress and the deposition of advanced glycation end products $^{(34)}$. In contrast, low-protein intake also exhibited a negative correlation with hearing discomfort based on mean hearing thresholds; however, only the hearing threshold at $1000 \mathrm{~Hz}$ exhibited a statistically significant correlation with protein intake. Some studies suggested that various types of peptides, such as insulin, orexin and leptin, played roles in hippocampal synaptic plasticity and enhancement of cognitive function ${ }^{(35)}$. Moreover, an animal study revealed that insufficient protein intake enhanced ototoxic side effects ${ }^{(36)}$. Therefore, it can be inferred that low-protein intake may have detrimental effects on neural function associated with auditory system.
There were some limitations in the present study. It is possible that the low-fat intakes observed in our sample were the consequence of other lifestyle risk factors, such as low socioeconomic status (SES), unemployment and poor hygiene ${ }^{(37,38)}$. Collinearity potentially occurs between different dietary habits and residual confounding factors, including both nutritional and non-nutritional confounders. Thus, it is possible that low SES, not a low-fat intake, could be related to hearing discomfort. To exclude this possibility, we analysed several potential confounding factors, including age, sex, BMI, economic status as represented by income level, smoking status and a medical history of hypertension, hyperlipidaemia and diabetes mellitus. Moreover, if a low SES or poor nutritional supply caused the hearing discomfort observed in this study, other nutritional factors, such as protein or energy intakes, would also show relationships with hearing. However, in this study, the total energy and protein intakes were not related to the hearing thresholds. A significant correlation between total energy intake and the hearing thresholds was observed in the unadjusted model but did not remain after the model was adjusted for covariates. Because nutritional needs differ according to age, sex and other covariates, it was reasonable to adjust the nutritional intakes according to these covariates. Our results did not reveal better or worse hearing thresholds according to the total energy intake, implying that the amount of fat consumed was not important for hearing. The proportional dietary intake of fat may be the factor related to hearing discomfort. Because the participants had an average total energy intake that was lower than the recommended value, it is also possible that the negative effects of the excessively high proportion of fat intake were outweighed by the positive effects of the increased fat intake on hearing function.

This study was based on a cross-sectional epidemiological survey, and it has some limitations that prevent an explanation of the causal relationships between the nutritional intake and hearing discomfort in the elderly. The cross-sectional nature of the study precluded an investigation of the effect of nutritional factors on the progression of hearing discomfort over time. Moreover, a possible reverse causality issue could not be excluded. The low-fat intake of our study population might be attributed to the rice-based Korean food culture, which differs from that of western countries. Therefore, the impact of a low-fat intake in this study cannot be generalised to other populations with different dietary cultures. Because a survival bias could affect hearing outcomes, people older than 80 years of age were not included in this study. Although the fidelity of our data was ensured by using masspopulation data based on the representative sampling of the KNHANES, selection bias remains possible in the present study. Finally, because this study was based on a survey that employed the complete $24 \mathrm{~h}$ recall method, some level of recall bias is inevitable.

Animal studies assessing the effects of low-fat diets and molecular biological techniques may be needed to elucidate the molecular mechanisms underlying the hearing-protective effects of dietary fats. Our findings provide clues regarding the potential mechanisms that underlie diet-related hearing discomfort and suggest possible strategies for preventing hearing discomfort through dietary modifications. Clinicians should evaluate patients' nutritional intake, both to prevent the 
development of other metabolic or cardiovascular disorders and to potentially facilitate hearing preservation.

The current study revealed that low-fat and low-protein diets are associated with increased hearing thresholds in the elderly Koreans. Given that total energy intake was not associated with hearing discomfort, the amount of dietary fat or protein is not important for prevention of hearing discomfort in the elderly Koreans; rather, the proportion of each nutrient among the total energy intake is key for prevention.

\section{Acknowledgements}

The authors gratefully acknowledge survey participants and examiners of the Division of Chronic Disease Surveillance in Korea Centers for Disease Control \& Prevention for participating in this survey and the dedicated work they provided.

This work was supported by a Research Grant funded by Hallym University Sacred Heart Hospital (HURF-2015-31).

H. G. C. designed the study. H. G. C. and S. S. performed statistical analysis. H. G. C. and S. Y. K. wrote the paper. All authors reviewed and approved the final article.

The authors have no conflicts of interest.

\section{References}

1. Department of Nutrition for Health and Development (NHD) of the World Health Organization (2008) Interim summary of conclusions and dietary recommendations on total fat \& fatty acids. http://www.who.int/nutrition/topics/FFA_summary_ rec_conclusion.pdf (accessed December 2014).

2. Honors MA, Harnack LJ, Zhou X, et al. (2014) Trends in fatty acid intake of adults in the Minneapolis-St Paul, MN Metropolitan area, 1980-1982 through 2007-2009. J Am Heart Assoc 3, e001023.

3. June S, Eva Erber O, Zhaohui C, et al. (2015) US adults recommended for weight reduction by 1998 and 2013 obesity guidelines, NHANES 2007-2012. Obesity 23, 527-531.

4. Gadgil MD, Anderson CA, Kandula NR, et al. (2015) Dietary patterns are associated with metabolic risk factors in south asians living in the United States. J Nutr 207753, 5.

5. Cree MG, Newcomer BR, Katsanos CS, et al. (2004) Intramuscular and liver triglycerides are increased in the elderly. J Clin Endocrinol Metab 89, 3864-3871.

6. Howard BV, Van Horn L, Hsia J, et al. (2006) Low-fat dietary pattern and risk of cardiovascular disease: the Women's health initiative randomized controlled dietary modification trial. JAMA 295, 655-666.

7. Schwingshackl L \& Hoffmann G (2014) Comparison of the long-term effects of high-fat v. low-fat diet consumption on cardiometabolic risk factors in subjects with abnormal glucose metabolism: a systematic review and meta-analysis. Br J Nutr 111, 2047-2058.

8. Schwab U, Lauritzen L, Tholstrup T, et al. (2014) Effect of the amount and type of dietary fat on cardiometabolic risk factors and risk of developing type 2 diabetes, cardiovascular diseases, and cancer: a systematic review. Food Nutr Res $\mathbf{5 8}$ (epublication 10 July 2014).

9. Miyake Y, Sasaki S, Yokoyama T, et al. (2006) Risk of postpartum depression in relation to dietary fish and fat intake in Japan: the Osaka Maternal and Child Health Study. Psychol Med 36, 1727-1735.

10. Sanchez-Villegas A \& Martínez-González MA (2013) Diet, a new target to prevent depression? BMC Med 11, 3 .
11. Liu JJ, Green P, John Mann J, et al. (2014) Pathways of polyunsaturated fatty acid utilization: Implications for brain function in neuropsychiatric health and disease. Brain Res 1597C, 220-246.

12. Gopinath B, Flood VM, Rochtchina E, et al. (2010) Serum homocysteine and folate concentrations are associated with prevalent age-related hearing loss. J Nutr 140, 1469-1474

13. Gopinath B, Flood VM, McMahon CM, et al. (2011) Dietary antioxidant intake is associated with the prevalence but not incidence of age-related hearing loss. J Nutr Health Aging 15 , 896-900.

14. Kang JW, Choi HS, Kim K, et al. (2014) Dietary vitamin intake correlates with hearing thresholds in the older population: the Korean National Health and Nutrition Examination Survey. Am J Clin Nutr 99, 1407-1413.

15. Gopinath B, Flood VM, Teber E, et al. (2011) Dietary intake of cholesterol is positively associated and use of cholesterollowering medication is negatively associated with prevalent age-related hearing loss. J Nutr 141, 1355-1361.

16. Evans MB, Tonini R, Shope CD, et al. (2006) Dyslipidemia and auditory function. Otol Neurotol 27, 609-614.

17. Jones NS \& Davis A (2000) A retrospective case-controlled study of 1490 consecutive patients presenting to a neurootology clinic to examine the relationship between blood lipid levels and sensorineural hearing loss. Clin Otolaryngol Allied Sci 25, 511-517.

18. Jones NS \& Davis A (1999) A prospective case-controlled study of 197 men, 50-60 years old, selected at random from a population at risk from hyperlipidaemia to examine the relationship between hyperlipidaemia and sensorineural hearing loss. Clin Otolaryngol Allied Sci 24, 449-456.

19. Gopinath B, Flood VM, Rochtchina E, et al. (2010) Consumption of omega-3 fatty acids and fish and risk of age-related hearing loss. Am J Clin Nutr 92, 416-421.

20. Kwon YS \& Ju SY (2014) Trends in nutrient intakes and consumption while eating-out among Korean adults based on Korea National Health and Nutrition Examination Survey (1998-2012) data. Nutr Res Pract 8, 670-678.

21. Paik HY (2008) Dietary reference intakes for Koreans (KDRIs). Asia Pac J Clin Nutr 17, Suppl. 2, 416-419.

22. Institute RRD (2006) Food Composition Table, 7th ed. Suwon, Korea: Rural Resource Development Institute.

23. The Korean Nutrition Society (2010) Dietary Reference Intakes for Koreans 2010, 1st ed. Seoul, Korea: The Korean Nutrition Society.

24. Korean Nutrition Society (2010) Dietary reference intakes for Koreans (KDRIs). http://www.kns.or.kr/FileRoom/FileRoom_ view.asp? mode $=$ mod\& restring $=\% 252$ FFileRoom $\% 252$ FFile Room.asp\% 253Fxsearch\%253D0\%253D\%253Dxrow\%253D 10\%253D\%253DBoardID\%253DKdr\%253D\%253 Dpage\%253 D1\&idx $=21 \&$ page $=1 \&$ BoardID $=$ Kdr\&xsearch $=1 \&$ cn_search (accessed December 2014).

25. Mathers C, Smith A \& Concha M (2003) Global Burden of Hearing Loss in the Year 2000, pp. 1-30. Global Burden of Disease 2000. Geneva: WHO. http://www.who.int/healthinfo/ statistics/bod_hearingloss.pdf (accessed December 2014).

26. Kim W, Fan YY, Barhoumi R, et al. (2008) n-3 Polyunsaturated fatty acids suppress the localization and activation of signaling proteins at the immunological synapse in murine CD4+ T cells by affecting lipid raft formation. J Immunol 181, 6236-6243.

27. Luchtman DW \& Song C (2013) Cognitive enhancement by omega- 3 fatty acids from child-hood to old age: findings from animal and clinical studies. Neuropharmacology 64, 550-565.

28. Lu JY, Huang KC, Chang LC, et al. (2008) Adiponectin: a biomarker of obesity-induced insulin resistance in adipose tissue and beyond. J Biomed Sci 15, 565-576. 
29. Hwang JH, Hsu CJ, Liu TC, et al. (2011) Association of plasma adiponectin levels with hearing thresholds in adults. Clin Endocrinol (Oxf) 75, 614-620.

30. Lavie CJ, Milani RV \& Ventura HO (2009) Obesity and cardiovascular disease: risk factor, paradox, and impact of weight loss. J Am Coll Cardiol 53, 1925-1932.

31. Li CM, Zhang X, Hoffman HJ, et al. (2014) Hearing impairment associated with depression in US adults, National Health and Nutrition Examination Survey 2005-2010. JAMA Otolaryngol Head Neck Surg 140, 293-302.

32. Connor WE (2000) Importance of $n-3$ fatty acids in health and disease. Am J Clin Nutr 71, Suppl., 171S-175S.

33. Torre P 3rd, Cruickshanks KJ, Klein BE, et al. (2005) The association between cardiovascular disease and cochlear function in older adults. J Speech Lang Hear Res 48, 473-481.
34. Fukui M, Kitagawa Y, Nakamura N, et al. (2004) Idiopathic sudden hearing loss in patients with type 2 diabetes. Diabetes Res Clin Pract 63, 205-211.

35. Marmot M (2005) Social determinants of health inequalities. Lancet 365, 1099-1104.

36. Lautermann J \& Schacht J (1995) Reduced nutritional status enhances ototoxicity. Laryngorbinootologie $\mathbf{7 4}$, 724-727.

37. Fadel JR, Jolivalt CG \& Reagan LP (2013) Food for thought: the role of appetitive peptides in age-related cognitive decline. Ageing Res Rev 12, 764-776.

38. Kumar S, Dagli RJ, Mathur A, et al. (2008) Oral hygiene status in relation to sociodemographic factors of children and adults who are hearing impaired, attending a special school. Spec Care Dentist 28, 258-264. 